\title{
Demand side load management using a three step optimization methodology
}

\author{
V. Bakker, M.G.C. Bosman, A. Molderink, J.L. Hurink, G.J.M. Smit \\ University of Twente \\ Department of Electrical Engineering, Mathematics and Computer Science \\ P.O.Box 217, 7500 AE, Enschede, The Netherlands \\ v.bakker@utwente.nl
}

\begin{abstract}
-
In order to keep a proper functional electricity grid and to prevent large investments in the current grid, the creation, transmission and consumption of electricity needs to be controlled and organized in a different way as done nowadays. Smart meters, distributed generation and -storage and demand side management are novel technologies introduced to reach a sustainable, more efficient and reliable electricity supply. Although these technologies are very promising to reach these goals, coordination between these technologies is required. It is therefore expected that ICT is going to play an important role in future smart grids. In this paper, we present the results of our three step control strategy designed to optimize the overall energy efficiency and to increase the amount of generation based on renewable resources with the ultimate goal to reduce the $\mathrm{CO}_{2}$ emission resulting from generation electricity. The focus of this work is on the control algorithms used to reshape the energy demand profile of a large group of buildings and their requirements on the smart grid. In a use case, steering a large group of freezers, we are able to reshape a demand profile full of peaks to a nicely smoothed demand profile, taking into the account the amount of available communication bandwidth and exploiting the available computation power distributed in the grid.
\end{abstract}

\section{INTRODUCTION}

In the last decades, more and more stress is put on the electricity supply and infrastructure. On the one hand, electricity usage increased significantly and became very fluctuating. Demand peaks have to be generated and transmitted, defining the minimal requirements in the supply chain. Thus, due to the fluctuating demand, minimal grid requirements have increased. Another effect of fluctuations is a decrease in generation efficiency [1].

On the other hand, reduction in the $\mathrm{CO}_{2}$ emissions and introduction of generation based on renewable sources are important topics today. Unfortunately, natural resources are dependent on very fluctuating and uncontrollable sun-, waterand windpower. Although there is some similarity in the energy demand pattern and the production pattern via renewable resources, they are not equal. For this reason, supplemental production is required to keep the demand and supply in balance, resulting in an even more fluctuating generation pattern for the conventional power plants. Finally, the introduction of new, energy efficient technologies such as electrical cars can result in a even further fluctuating electricity demand. Uncontrolled charging of electrical cars will result in a high demand of electricity since these vehicles need to be charged fast to ensure enough capacity for the upcoming trip. Lowering the peaks in demand is desirable to prolong the usage of the available grid capacity.

A possible solution for these problems may be to transform domestic consumers from static consumers into active players in the optimization process. More and more new technologies with controllable load and generation are developed, such as controllable white goods and micro-generation. Furthermore, domestic energy storage of both heat and electricity is becoming quite common. The introduced flexibility in control of these techniques results in a optimization potential. The goal of our research is to determine a methodology to use this optimization potential to 1) optimize efficiency of current power plants, 2) support large penetration level of renewable sources (and thereby facilitate the means that are needed for $\mathrm{CO}_{2}$ reduction) and 3) optimize usage of the current grid capacity.

In [2] a control strategy is presented to exploit this optimization potential in a generic way. The methodology is flexible in both the optimization objective and the technologies available within houses. This control strategy consists of three steps.

In the first step, a system located at the consumers predicts the production and consumption pattern for all appliances for the upcoming day. For example, in a normal household multiple appliance like a tv, washing machine, central heating are present. For each appliance, based on the historical usage pattern of the residents and external factors like the weather, a predicted energy profile is generated. The combination of these energy profiles determines the optimization potential of all appliances located in the house.

In the second step, these optimization potentials can be used by a central planner to exploit the potential to reach a global objective. Example objectives are peak shaving or compensating the fluctuation of the production of renewable sources like windparks. The result of the second step is a planning for each household for the upcoming day.

In the final step, a realtime control algorithm decides at which times appliances are switched on/off, when and how much energy flows from or to the buffers and when and which generators are switched on. This realtime control algorithm uses steering signals from the global planning as input, but preserves the comfort of the residents in conflict situations. In this way, the algorithm can also deal with prediction errors.

The first and third step are already studied quite well and the results are presented in [3] and [4] respectively. The math- 
ematical principles of the second step are also investigated [5], but no actual implementation was made yet. Therefore, this paper focuses on the planning algorithm, the way the steering signals for the planning are determined, the required hierarchical communication structure and the amount of data transferred. The different versions of the hierarchical planning algorithm are implemented in our simulator [6] to analyze the planning results and data transferred.

The rest of this paper is structured as follows. First the different approaches for determining and distributing the steering signals are described. Next, a comparison with other state-ofthe-art research is made and the differences with our approach are pointed out. In Section IV a use case is described and Section $\mathrm{V}$ describes the algorithms. The results are presented in Section VI and in the last section some conclusions are drawn and future work is given.

\section{APPROACH}

Key in optimization methodologies is communication between all the different technologies. This communication takes place at different levels, from short-range communication in house between appliances and the domestic controller to widerange communication between neighborhood control nodes and a central planner. The simplest case of communication in house is the wire connecting the thermostat and the (High Efficiency) boiler. However, smart appliances and Demand Side Load Management require a connection between the domestic controller and these smart appliances.

Besides communicating within the house, the optimization methodologies need communication links with parties in the grid to work towards a global objective. Although communication technologies are still improving, they still have limited bandwidth and introduce latencies during communication. A control strategy should take these restrictions into account.

Besides limited communication, also only a limited amount of computational resources is available. Since finding an (near) optimal utilization of the available resources in the smart grid requires a lot of computational power [5], a control scheme must exploit the computational power spread in the smart grid as much as possible. For this reason, our planning and control methodology is organized in a tree structure. The root node of the tree is the global planner. The global planner tries to optimize the energy profile of the whole fleet, based on a given objective. Taking into account the information on the predicted energy profiles of all consumers, this given objective is transformed into a desired energy profile of the fleet. Since matching this desired energy profile exactly is very difficult or even impossible, the desired energy profile is described by a lower and upper bound. The aggregated load profile of all buildings steered by the planner should fall within these bounds.

The root planner tries to achieve this profile by decomposing the profile in subparts and delegating these parts over planners located in the nodes of the hierarchical structure directly below him. Each planner directly below the root planner is responsible for planning its part of the tree such that his share of the global profile is reached. Again, these planers try to achieve this goal by delegating subparts of their desired profile to their children. The planner on the bottom of the tree is directly connected to the controllers located in the houses. It tries to achieve his given energy profile by sending steering signals to the domestic controllers. Based on these steering signals, the domestic controllers generate a planning for the coming day and send this planning to connected the planner. On every level, the data is aggregated and sent further upward in the tree. Based on the mismatch between the planning and the desired profile, the central node adjusts the partition of the profile, sends this to the planners directly below him and then the process starts again. This iterative process is organized such that after several iterations, the resulting profile falls between the lower and upper bound (if possible).

Using such an approach exploits the computational power available within the grids. Due to the tree structure, the required amount of communication is reduced. This fits well within the structure of smart grids, where a fast communication link between buildings and the nearby planner is available. Higher level planners can be further away, connected with less advanced communication links since the amount of information that needs to be exchanged reduces significantly.

\section{RELATED WORK}

Most approaches found in literature are hierarchically structured, agent based solutions. The hierarchical structure ensures the scalability of the solution. Although a lot of approaches claim to be distributed without a central algorithm, all approaches found have one decision-making element (node, agent, etc.).

The methodology described in [7] is a decentralized, agentbased, realtime control methodology. On different levels in the network agent-based nodes are installed controlling and/or monitoring a network element (e.g. house appliances, capacitor banks, substation voltage). These nodes communicate with each other to exchange information, optionally also with human operators. The communication is structured in a sort of hierarchical way: the nodes are divided in groups exchanging information with each other. When all information is available, the group leader makes a decision, optionally in cooperation with other group leaders.

The PowerMatcher described in [8], [9] is an agent-based, hierarchical optimization methodology. Every appliance in the house can be controlled by an agent. This agent sends a bid (amount of demand/generation and a price) to the agent one level higher (house agent), which aggregates all bids and sends the aggregated bids one level higher, etc. The root agent decides, based on the bids and the objective, the market clearing price. This price is distributed and each agent knows what to do based on the bid made and the price. The agents coupled to an appliance can use predictions to optimize their bidding strategy. However, this is on a local (appliance) level, only leading to profit optimization of the appliance-agent itself.

For the GridWise project [10] a decentralized control methodology with dynamic pricing is used. In this approach, no centralized algorithm is used. Field tests showed that the dynamic pricing can reduce peaks up to $15 \%$.

In [11] a methodology to manage thermal household appliances (fridge, freezer, boiler) is described. Thermal household 


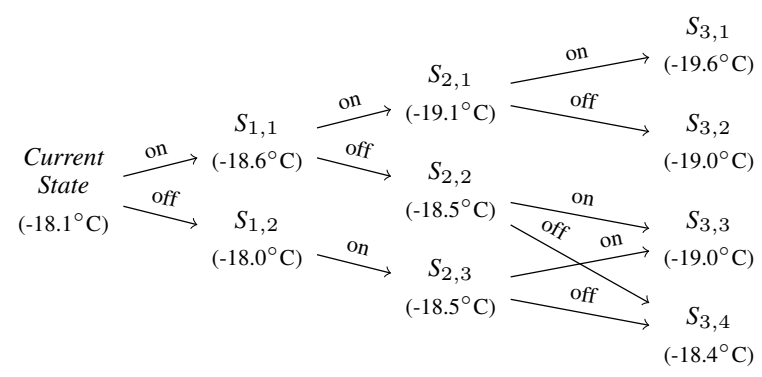

Fig. 1. Planning of a freezer

appliances can have a buffering property: cooling a freezer during low demand periods to prevent having to cool during peak demand periods. This approach uses one central controller, all houses are directly connected to this controller. To prevent sending too much detailed information (privacy), only the costs to switch an appliance on or off are sent. The central controller decides how much appliances should be switched on and determines the switch-on-price and switch-off-price.

The similarities between the described approaches and our approach reflect in the control up to an appliance level and the hierarchical structure with aggregation on each level. The main differences are the prediction/planning and the lack of agents. Although the PowerMatcher approach uses prediction and planning on a device level, this is utilized for profit raising of the agent itself. The latter is also the main difference between our approach and an agent-based approach: agents are greedy and try to optimize their own profit where our optimization methodology tries to reach a global objective for the whole fleet. Furthermore, our approach uses variable steering signals instead of the same price/signal for everyone and has a larger planning horizon (up to one day in advance).

\section{USE CASES}

To analyze the potential of our approach, we try to control a large group of freezers located in buildings as an use case. The internal temperature of freezers has to be maintained between certain limits. Since the environment of a freezer is relatively static, freezers show a very regular and predictable pattern. When the cooling element of a fridge is switched off the temperature rises slowly. Once a certain upper temperature is reached, the cooling element is switched on to cool to a certain lower temperature threshold. By advancing and postponing the switching points of the cooling element, the energy profile of the freezer can be altered, respecting the temperature boundaries.

The regular pattern of the freezer simplifies the planning of the device. The goal of the planner is to stay within the temperature limits, while reducing the overall costs. These costs can be determined by electricity prices, but also by steering signal (which can vary over the day) from the global planner. By using a dynamic programming approach, of which some states are depicted in Figure 1, an optimal planning for a freezer can be obtained. Each state change requires a certain amount of electricity and leads to a certain cost. Goal of the planner is to minimize costs.

To analyze the performance of our algorithm, it is implemented in our simulator. Two scenarios are simulated. The first scenario is a simulation consisting of 50 houses (with the above described freezer). This scenario can be used to analyze the performance of the planner cooperating with the house controllers. Here, only one structure layer is required, since planning 50 houses is still feasible. Using only one planner, the effect on the energy profile of the whole group by using different steering signals can be studied.

In the second scenario, 500 houses are controlled. To control such a large group, a hierarchical approach is required. Again, different steering signals can be used to study the effect on the whole group, e.g. multiple ways to divide the whole planning can be tested.

\section{Algorithm}

The above mentioned case uses a set of individual houses containing a freezer. In this section we define a global objective on this use case and present a heuristic that produces local day schedules to which the individual freezers must comply as much as possible. First we explain the objective. Next we introduce the heuristic and the choice to use local planning techniques in addition to variable prices (for different houses).

The global objective of the use case is to spread the electricity consumption of the whole fleet equally over the planning horizon, which is one day. The general idea is that, suppose we are able to consume this flat line, this means that this electricity can be produced more efficiently, since constant generation is preferred over adaptive (fluctuating) generation. For the planning the time is descretisized into $N R$ intervals. Given a fleet of $H$ houses, first the total amount of electricity $T E C$, which all houses together would consumed based on their individual planning, is calculated. So TEC denotes the planned consumption of electricity, if each house optimizes the use of its freezer for his own benefit. We define the prefered consumption $P$ to be equal to this planned consumption per period, i.e. $P:=\frac{T E C}{N R}$. So we want to steer towards a total consumption, which corresponds to the amount which results from optimal individual planning. For each individual house this means that the operational behavior, once guided from a global perspective, remains similar in the sense that the total electricity consumption is equivalent to the consumption in standalone operation. The difference lies in the fact that for each interval an average consumption $P$ is required on the complete set of houses. Since reaching exactly $\mathrm{P}$ might be impossible, we allow the expected consumption in each interval to be in the band $[0.8 P, 1.2 P]$.

As a measure to analyze the quality of a planning, we define the following. Let $T P L_{j}$ be the planned consumption in interval $j$ following from our applied heuristic, then $M_{j}:=\max \left\{T P L_{j}-1.2 P, 0.8 P-T P L_{j}, 0\right\}$ denotes the measure of quality for this interval. Now $M=\sum_{j=1}^{N R} M_{j}$ is the measure of quality for the heuristic, which is to be minimized.

The base of the heuristic for house $i$ is a local dynamic program $D P^{i}$ that solves the use of a freezer to optimality, only regarding local constraints (the operational characteristics 
of the freezer determine the possible states) and a price vector $p^{i}$ :

$$
D P^{i}\left(p^{i}\right) \rightarrow P L^{i}
$$

where $P L^{i}$ is the resulting planning vector. This price vector $p^{i}$ denotes the costs of using the freezer in the intervals. The prices have to be related to the energy cost price, but they are also used to steer the profile to the desired profile. As a consequence, the costs are determined by two parts: electricity costs $e p^{i}$ and artificial costs $a p^{i}$. In the use case the electricity costs remains constant during the different iterations of the planning process. The artificial costs are used to move the consumption of the freezer to specific periods. Summarizing, we use as 'interface' to the local planning done by the dynamic program the artificial costs $a p^{i}$. The planned consumption $T P L$ of the whole fleet is an aggregation of the local plan $P L^{i}$ resulting from the dynamic programs $D P^{i}$ :

$$
T P L=\sum_{i=1}^{H} P L^{i}=\sum_{i=1}^{H} D P^{i}\left(p^{i}\right) .
$$

Solving the use case to a global optimum means that an exploration of the price space of all price vectors for all houses is needed. The power of the local dynamic program is that it produces a fast solution for a local problem, instead of applying a dynamic program approach to the complete set of houses simultaneously. The proposed heuristic uses this fast local approach iteratively to find a solution for the complete set of houses. However, the number of iterations needs to be limited to gain as much as possible from this advantage of having a fast local procedure.

In general the iterative search works as follows. The initial price vector is set to the electricity prices. Dependent on the outcome of the (initial) planning the prices are changed based on the artificial price $a p^{i}$. Initially, these $a p^{i}$ are also set to the electricity prices.

$$
\begin{gathered}
p^{i, 1}=e p^{i}, \\
a p^{i, 1}=e p^{i} .
\end{gathered}
$$

If after the planning in iteration $n$ the consumption of house $i$ in is too high for a certain interval, the price for this interval is enlarged based on the deviation from the target planning and the previous artificial price $a p^{i}$. The ensure stabilization of the algorithm, the resulting increases will get smaller with the increasing number of iterations. More formally, the prices for iteration $n$ and house $i$ are changed via:

$$
\begin{array}{r}
p^{i, n}=\operatorname{norm}\left(p^{i, n-1}+\operatorname{addPrice}\left(a p^{i, n-1}, T P L^{n-1}\right)\right), \\
a p^{i, n}=a p^{i, n-1}-\frac{p^{i, 1}}{i t},
\end{array}
$$

where $i t$ is the maximum number of iterations, $T P L^{n}$ is the planned total consumption in iteration $n$ and $\operatorname{norm}()$ is a normalizing function (so the overall costs do not rise).

A first variant of the heuristic uses similar price vectors for all houses. Here addPrice $\left(a p^{i, n-1}, T P L^{n-1}\right)$ returns a vector $\left(a_{1}, \ldots, a_{N R}\right)$, where:

$$
a_{j}= \begin{cases}a p_{j}^{i, n-1} & \text { if } T P L_{j}^{n-1}>1.2 P \\ 0 & \text { otherwise }\end{cases}
$$

Note that addPrice is nonnegative, meaning that we want to shift peak consumption to other intervals by only increasing the prices in peak intervals. To prevent overcompensating behavior, we only adapt the prices for above-average consumption and not for below-average consumption.

In the first approach the search space is limited since we use a equal price for all houses. In a second variant we create diversity between houses by allowing only a fraction of he houses to change its price. The vector $\left(a_{1}, \ldots, a_{N R}\right)$ resulting from addPrice is then defined as follows:

$$
a_{j}= \begin{cases}a p_{j}^{i, n-1} & \text { if }\left(T P L_{j}^{n-1}>1.2 P\right) \text { AND } \\ & \quad\left(\operatorname{rand}(0,1)^{i}<\frac{M_{j}^{n-1}}{T P L_{j}^{n-1}}\right) \\ 0 & \text { otherwise, }\end{cases}
$$

where $\operatorname{rand}(0,1)^{i}$ is a random number between 0 and 1 for house $i$.

Within the hierarchical structure we can implement the second variant in two different ways. These two ways differ where the stochastic choice of adding a price is made. On the one hand, the random choice to add an additional artificial cost in a certain interval can be picked at the bottom of the hierarchical structure, i.e. at the house controller. On the other hand, we may also pick this choice at the first branch in the structure, meaning that all underlying houses get the same price vectors. This can be seen as a compromise between the idea of the first and second variant, allowing only different prices on a higher level in the structure.

The heuristic can use different stop criteria. In the current (initial) implementation the heuristic ends when the maximum number of iterations it is reached to analyze the impact of the algorithm in each iteration. In future work, each planner within the structure can end the search in its substructure whenever the aggregated planning of this substructure confirms the desired planning for this planner or if there has been no significant improvement in the last few iterations.

\section{RESUlts}

As described earlier, two use cases are simulated. First, the different approaches in distributing the price vectors are described. Another important aspect is the required communication, which is analyzed in subsection VI-B.

\section{A. Impact of different price vectors}

As described above, there are different possibilities a) how price vectors are determined and b) how they are distributed over different branches of the tree. In the simplest case, there is only one global planner and the house controllers directly cooperate with this global planner. This is the case in the first simulation, where 50 houses are simulated. The freezers in the houses all start with different starting temperatures and have different characteristics, i.e. there are differences in cooling capacity and the insulation quality.

Using the prices determined by Eq. (5), (6) and (7) results in uniform price vectors which are sent to all houses. In other words, the prices are the same for every house. The results of the planning using this approach is depicted in Figure 2(a). In this figure, the evolution of the energy profile can be seen. 


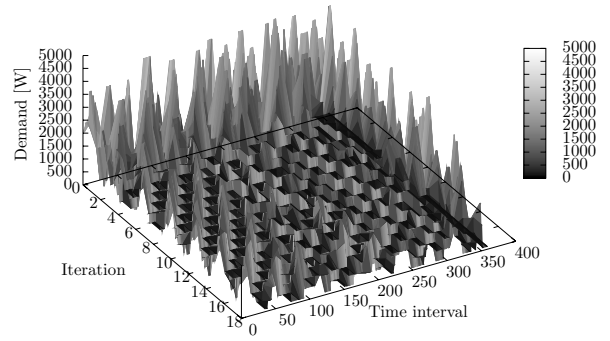

(a) Using uniform price vectors

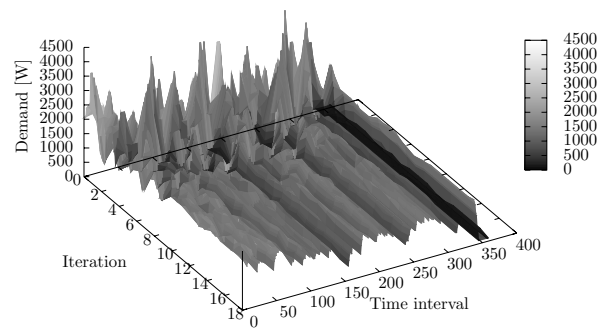

(b) Using different price vectors

Fig. 2. Results of planning for 50 houses

In the first iteration, the price vector contains only a single price for all time intervals. This results in a typical cyclic load, which aggregated leads to large peaks in demand. Since all house controllers receive the same price vector in the next iterations, they all try to shift their cooling cycles in the time interval with low costs, resulting an alternating pattern of consumption profiles.

When using different prices for each house controller, as described by Eq. (8), the quality of the planning improves significantly. The result of this scheme is depicted in Figure 2(b). On the one hand, the production pattern is far more flattened (as asked for by the global objective). On the other hand, this flattened production pattern is already reached after roughly ten iterations. Due to the reduced correction in price vector in the last iteration, the planning does not alter as much as in the beginning (see the minor differences between iteration 10 and 19 in Figure 3). Although the production pattern is quite flat, the global objective is not reached for every time interval. This is caused by the limited amount of freezers. Although each freezer has a different state, the number of state combinations of the group is limited.

When 500 houses are simulated, the number of possibilities increases significantly. It is then expected that a more flattened production pattern can be reached. The planning of the 500 houses is organized in a tree structure. One possibility to alter the price vectors is to use different price vector for a whole branch of the tree. This scheme is used in the planning depicted in Figure 4(a). Here, the prices are adjusted with a certain probability only at the top of the tree.

If a price for a branch has changed, this has an impact on the whole subtree. As expected, due to the different pricing scheme for subtrees within the whole tree, a more flattened production profile is obtained. However, since still a large group of house controllers obtain the same price vector,

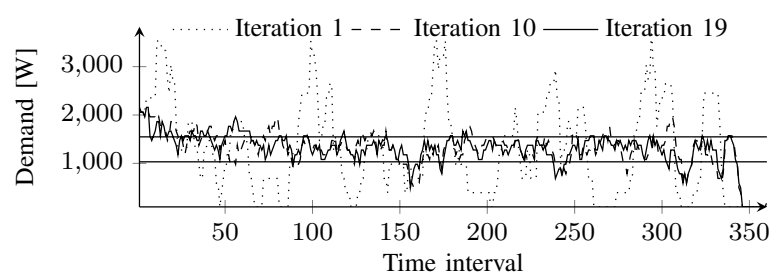

Fig. 3. Demand profile of after multiple iterations for 50 houses, different price vectors

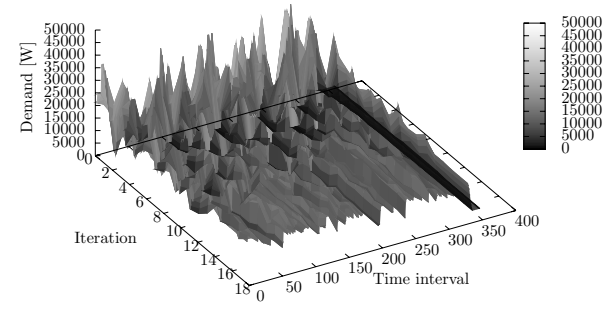

(a) Using different vectors at the top

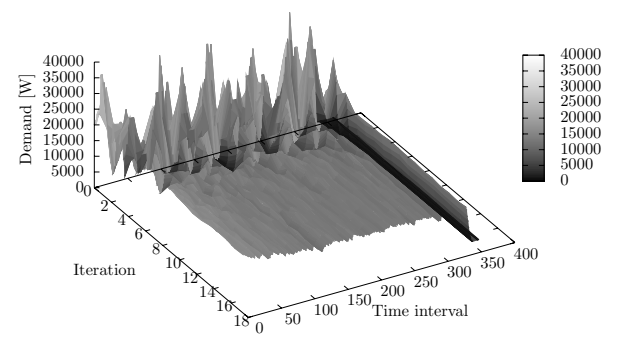

(b) Using different vectors at the bottom

Fig. 4. Results of planning for 500 houses

between the fourth and eighth iteration still a lot of shifts in load can be seen. Since the alternation in prices is moderated, eventually a flattened profile is reached.

The most advanced scheme is to distribute the load evenly over the tree and let the bottom planning determine the chances to accept the prices vector. The top planner here determines the global objective, i.e. the demand profile for each subtree. Each planning below then distributed its part of the profile to its children recursively. The bottom planners, communicating with the house controllers, try to reach this profile using the approach described by Eq. (8). Once the bottom planners are satisfied with the result for their subproblem, this is communicated upwards the tree. If the global objective is not reached, another distribution of the production pattern over the tree is possible. Using such an approach, the most variation in price vector is obtained. As can be seen in Figure 4(b), this leads to the best results both in the amount of required iterations as in the end result of the planning. Here, $M$ drops from 2,076,602 to 270,700 almost linearly in six iterations. After the sixth iteration M reduces (almost linearly) to 197,174.

Note that in all figures a sharp drop in demand in the last time intervals can be seen. This is a short-term effect since the house controllers all let the temperature rise as high as possible, resulting in the lowest cost. 


\section{B. Communication}

Due to the tree like structure, the amount of communication is reduced significantly. When dividing all the buildings over the tree structure, the tree should be as wide as possible in the bottom of the tree and as narrow as possible in the top. Using this structure, the algorithms can be distributed to the bottom of the tree as much as possible, exploiting the available computation power. Furthermore, it is assumed that house controllers and the bottom planners are close to each other with good communication links, resulting in fast communication. Going further up the tree the amount of communication is reduced, which unifies with the fact that communication links between higher planner can have less bandwidth and higher latency.

Since in our approach only price vectors and production patterns are used, the amount of information that needs be exchanged is limited. For each time interval to be scheduled, only a single number needs to be transmitted. Since a production or a price can be represented by a single number, the message size between two entities is determined by the planning horizon (the amount of time intervals to be planned) and the representation of the numbers. In our simulation of 500 houses, a planning is made for each four minutes for a whole day, leading to 360 time intervals.

Since only small numbers are exchanged at the lower level planners, fewer bits are required at these levels. This is beneficial, since most data is transmitted at these levels. Going upwards in the three, the numbers increase and the amount of bits can be increased accordingly.

Using 16-bit integers at the lowest planners using our own developed protocol, messages are 725 Bytes. Such message sizes are acceptable with current communication technology. The planning generated in Figure 4(b) consisted of nineteen planning iterations on the bottom level and only one global iteration, required a total amount of $13 \mathrm{MB}$, of which $99.8 \%$ is transmitted at the bottom level. Although this seems quite a lot of data, note that a very fine grained planning is obtained for a whole day. Furthermore, many iterations were executed to analyze the effect of the algorithms. By planning for a time interval of fifteen minutes (similar to the electricity markets time intervals), the amount of data can already be reduced by a factor of four. Since the algorithm reaches a good result after only a few iterations, another reduction can be achieved by reducing the amount of iterations at the bottom planners. These numbers can be reduced even further by adding compression and optimizations in information encoding.

\section{CONCLUSION}

The result of the freezer use case show that our three step methodology is able to apply global optimization techniques for a large group of houses. Both the predictions and planning are performed at each house, exploiting the available distributed computational power available in the grid. Due to the subsequent division of the large optimization problems into subproblems via a tree structure, a fast scalable system is achieved.

Using uniform prices leads to a sort of worst case scenario. Since all individual house controllers try to minimize their own cost, they all optimize to periods with low costs, leading to a shift of demand peaks instead of the desired profile. In other words, addressing each house individually by using different steering signals gives the best results.

By choosing a proper tree structure, communication requirements can be kept low. The use of simple price and production patterns lead to small messages to be sent.

Although the initial version, as shown in this work, already shows promising results, improvements are still possible and needed. On multiple levels in the tree, better approaches to determine when to stop the planning are needed to reduce the amount of iterations required during planning. Furthermore, by optimizing the way information is encoded during communication, bandwidth requirements can further be reduced.

Furthermore, the improved algorithms can be extended to steer a larger fleet of different kind of appliances, including electrical cars and HVAC systems (consumers), microCHP appliances (producers) and heat stores/batteries (buffers). Although the same control strategy as presented in this work can be used, different kind of appliances react differently on changes in the price vector. This will influence the amount of required iterations.

\section{ACKNOWLEDGMENT}

This research is conducted within the SFEER project (07937) supported by STW, Essent and Gasterra.

\section{REFERENCES}

[1] A. de Jong, E.-J. Bakker, J. Dam, and H. van Wolferen, "Technisch energie- en CO2-besparingspotentieel in Nederland (2010-2030)," Platform Nieuw Gas, p. 45, Juli 2006.

[2] A. Molderink, V. Bakker, M. Bosman, J. Hurink, and G. Smit, "A threestep methodology to improve domestic energy efficiency," in IEEE PES Conference on Innovative Smart Grid Technologies, 2010.

[3] V. Bakker, M. Bosman, A. Molderink, J. Hurink, and G. Smit, "Improve heat demand prediction of individual households," in Conference on control methodologies and technology for energy efficiency, March 2010.

[4] A. Molderink, V. Bakker, M. Bosman, J. Hurink, and G. Smit, "Domestic energy management methodology for optimizing efficiency in smart grids," in IEEE conference on Power Technology. IEEE, 2009.

[5] M. Bosman, V. Bakker, A. Molderink, J. Hurink, and G. Smit, "On the microchp scheduling problem (accepted)," in Third Global Conference on Power Control and Optimization. IEEE, 2010.

[6] A. Molderink, M. Bosman, V. Bakker, J. Hurink, and G. Smit, "Simulating the effect on the energy efficiency of smart grid technologies (accepted)," in Winter Simulation Conference 2009. IEEE, 2009.

[7] P. Hines, S. Hamilton, R. Yinger, C. Varanian, A. Feliachi, and K. Schoder, "Integrated, agent-based, real-time control systems for transmission and distribution networks," Grid-Interop Forum 2007, Tech. Rep., 2007.

[8] M. Hommelberg, B. van der Velde, C. Warmer, I. Kamphuis, and J. Kok, "A novel architecture for real-time operation of multi-agent based coordination of demand and supply," in Power and Energy Society General Meeting - Conversion and Delivery of Electrical Energy in the 21st Century, 2008 IEEE, July 2008, pp. 1-5.

[9] M. Hommelberg, C. Warmer, I. Kamphuis, J. Kok, and G. Schaeffer, "Distributed control concepts using multi-agent technology and automatic markets: An indispensable feature of smart power grids," in IEEE Power Engineering Society General Meeting, 2007, 2007.

[10] D. Chassin and L. Kiesling, "Decentralized coordination through digital technology, dynamic pricing, and customer-driven control: The gridwise testbed demonstration project," The Electricity Journal, vol. 21, pp. 5159, 2008.

[11] S. Koch, M. Zima, and G. Andersson, "Active coordination of thermal household appliances for load management purposes," in IFAC Symposium on Power Plants and Power System Control, 2009. 